\title{
How Visualization Layout Relates to Locus of Control and Other Personality Factors
}

\author{
Caroline Ziemkiewicz, Member, IEEE, Alvitta Ottley, R. Jordan Crouser, Ashley Rye Yauilla, \\ Sara L. Su, Member, IEEE, William Ribarsky, Member, IEEE, and Remco Chang, Member, IEEE
}

\begin{abstract}
Existing research suggests that individual personality differences are correlated with a user's speed and accuracy in solving problems with different types of complex visualization systems. We extend this research by isolating factors in personality traits as well as in the visualizations that could have contributed to the observed correlation. We focus on a personality trait known as "locus of control" (LOC), which represents a person's tendency to see themselves as controlled by or in control of external events. To isolate variables of the visualization design, we control extraneous factors such as color, interaction, and labeling. We conduct a user study with four visualizations that gradually shift from a list metaphor to a containment metaphor and compare the participants' speed, accuracy, and preference with their locus of control and other personality factors. Our findings demonstrate that there is indeed a correlation between the two: participants with an internal locus of control perform more poorly with visualizations that employ a containment metaphor, while those with an external locus of control perform well with such visualizations. These results provide evidence for the externalization theory of visualization. Finally, we propose applications of these findings to adaptive visual analytics and visualization evaluation.
\end{abstract}

Index Terms-Visualization, individual differences, locus of control.

\section{INTRODUCTION}

G RAPHIC designs, such as diagrams and maps, J support users in processing sensory data, reasoning about it, solving problems, and identifying patterns [1]. A well-designed visualization accounts for both the complex preattentive and cognitive processes triggered when a user views it. The balance of visual elements can dramatically affect the user's comprehension of the information presented. As a result of separable and integral feature relationships, using several elements in conjunction can amplify or dampen a single element's effect [2]. Understanding the relations among preattentive processes, cognitive processes, and visual interfaces is a longstanding goal of the visual analytics community, but evaluating interfaces in this context is difficult for many reasons. One reason is the problem of individual differences.

Because visualizations are meant to support complex thinking, they may be more sensitive to nuances

- C. Ziemkiewicz is with the Department of Computer Science, Brown University.

E-mail: cziemki@cs.brown.edu

- A. Ottley, R.J. Crouser, and R. Chang are with the Department of Computer Science, Tufts University.

E-mail: alivttao@cs.tufts.edu, rcrous01@cs.tufts.edu, and remco@cs.tufts.edu

- A.R. Yauilla is with the Department of Computer Science, Winthrop University.

E-mail:yauillaa2@mailbox.winthrop.edu

- S.L. Su is with Google, Inc. E-mail:sara.su@google.com

-W. Ribarsky is with the Department of Computer Science, UNC Charlotte.

E-mail: ribarsky@uncc.edu of an individual user's cognitive style than other types of interfaces. This would explain findings that show significant differences in visualization performance based on personality. Earlier work has found an effect from the personality dimension known as "locus of control" [3], [4]. This dimension measures a person's tendency to see herself as either shaped by or in control of external events. While the effects of individual differences such as locus of control have been observed, an explanation for why those differences arise remains elusive. Early work in this area employed real-world data exploration systems that differed on many dimensions, including the use of color, labeling, interaction, and layout style. Any of these variables may contribute to the effect of locus of control, making it difficult to apply the findings to visualization design for different user groups.

We propose that layout style is the key variable that determines the interaction between locus of control and compatibility with different system designs. Our definition of layout encompasses any differences in the spatial arrangement and presentation of marks in a visualization. This is to be distinguished from differences in the visual encoding, that is, how individual data variables are mapped to individual graphical variables, such as color or size ${ }^{1}$.

Our hypothesis is that individual differences based on locus of control are more affected by layout in this sense than by visual encoding or interaction style.

1. In cases where a variable is directly mapped to spatial position, as in a scatterplot, that aspect of spatial arrangement is of course part of the encoding. No such mapping existed in the visualizations we study. 
This hypothesis is based on the idea that locus of control can provide insight into a user's tendency to rely on the external representations that a visualization employs. The overall arrangement of visual elements in a layout is more likely to directly affect the nature of this external representation than surface qualities such as color and shape, or more intangible qualities such as interaction style.

\subsection{Contributions}

This paper makes a number of contributions to our understanding of how personality factors affect how people use visualizations:

- First, we present experimental evidence to show that visual layout is a key factor in previous findings in individual differences. Our findings expand previous work by showing that the effect of locus of control can still be found when restricting visualization differences to layout factors.

- Second, we use these findings to argue for a model of visualization use based on a user's adoption of external representations. Our findings suggest that locus of control affects the use of different visualization types by affecting a user's willingness to adapt to a novel externalization of information. This framework places these findings in the context of external representation as a model for visualization use.

We evaluate our hypothesis in a study with 240 online subjects who varied in their locus of control. Participants are presented with four variations of a hierarchy visualization showing phylogenetic data (Figure 1). These designs include a view that employs a list-like organizational structure (V1), a view that presents the hierarchy in a strong containment metaphor (V4), and two designs that lie between these extremes (V2 and V3). We hypothesize that users with a more external locus of control are more willing to adapt their thinking to unfamiliar visual metaphors than those with an internal locus of control. We show how locus of control can predict performance on inferential task questions using these interface designs. Specifically, we test the hypothesis that an individual with a more internal locus of control will show a performance decrease when using layouts with a strong containment metaphor, while those with a more external locus of control will not show this decrease. Our findings provide evidence for the externalization model of visualization use and can inform the design of visualization interfaces adapted to an individual's needs.

\section{Related Work}

There is a substantial history of research in how individual differences affect interface use in the broader human-computer interaction field, reviewed by Dillon and Watson [5]. A subset of this research focuses specifically on individual differences in visualization, driven by the belief that a reliance on visual and spatial cognitive abilities makes individual differences a potentially greater factor in this field. We review findings on how cognitive differences affect visualization use and discuss why locus of control is a potentially significant factor.

\subsection{Individual Differences and Visualization}

Much of the existing work in the visualization field on the impact of individual differences has focused on the influence of perceptual ability on visualization use. Conati and Maclaren [6] find that a user's perceptual speed predicts whether a star graph or heatmap will be most effective. Similarly, Allen [7] finds a role for perceptual speed and spatial scanning ability in search performance. However, he found that users do not tend to optimize their visualization use for greater search efficiency, and proposes the development of user models to automatically guide users toward optimal strategies. This work shows that perceptual abilities can affect task performance, but suggests that the connection between these abilities and a user's cognitive strategies may be less direct.

Taking a more cognitive perspective, Chen [8] found that spatial ability has no effect on participants' search performance in a visualization of paper citation links. Cognitive factors did, however, play a role in users' subjective feedback; users with high associative memory rated the interface higher on usefulness, and users with high spatial ability rated the tasks higher on familiarity. Chen goes on to suggest that users with varying cognitive abilities may be more or less likely to impose certain kinds of mental models on data.

A related finding comes from Ziemkiewicz and Kosara [9], who studied the role of personality and spatial ability in a user's ease in switching between incompatible visual metaphors in tree visualization use. They found that users with high spatial visualization ability and a high score on the Openness scale of the Big Five Personality Inventory [10] were more adept at answering questions despite a verbal metaphor that conflicted with the visual metaphor of the visualization they were viewing. Both this work and Chen's suggest that users with different cognitive styles may be more or less comfortable with adapting their thinking to an external representation.

Tversky et al. [11], [12], [13] studied how individual differences in ability impact the extraction of structure and function from diagrams. They found that participants with high ability form mental models integrating structure and function, while those with low ability form models dominated by structure. They suggest that structural diagrams designed for the latter group can be annotated with functional information for improved comprehension [14].

These findings have begun to build the case that some individual differences in visual analytics can be 


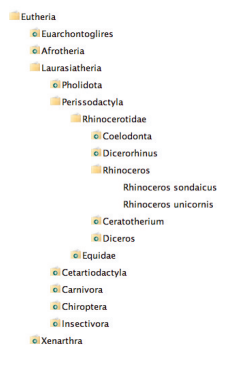

(a) V1: Basic Tree

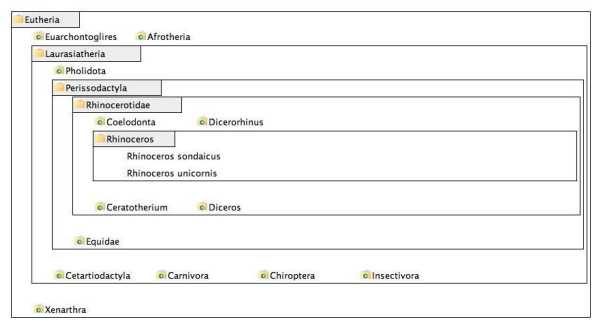

(c) V3: Indented Boxes

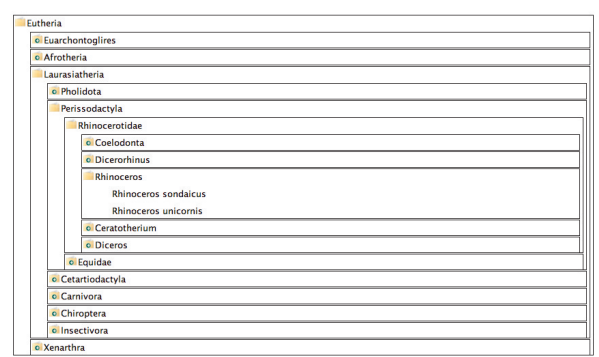

(b) V2: Bordered Tree

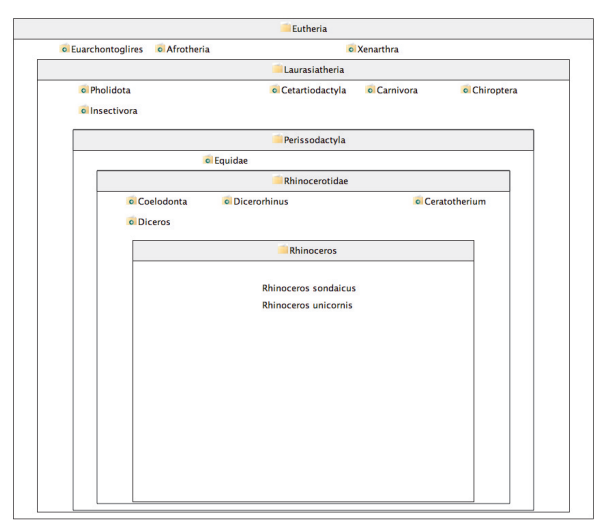

(d) V4: Nested Boxes

Fig. 1. The four visualizations used in our study. Each view is showing the same portion of one of the phylogenetic tree datasets.

attributed to a complex interaction between the mental model suggested by a visual layout and the user's own cognitive style. However, most previous studies that show an effect of individual differences either examine a single system or compare two or more systems with numerous differences. In order to apply knowledge of individual differences to visualization design, it is necessary to make a clearer connection between personality groups and exactly which factors lead them to better performance with one visualization over another. Context for these connections can come from psychology research on how personality relates to problem solving and decision making.

\subsection{Personality and Problem Solving}

Problem solving is the process by which we bridge the gap between the perceived and a desired outcome. Since this process often requires complex thought, researchers have long been investigating the effect of personality on problem solving and decision making processes. These investigations have identified a series of personality traits that impact problem solving including extraversion, neuroticism and locus of control. For example, when approaching a problem, persons who score high on the judgment scale of the MyersBriggs Personality Inventory (i.e., those who are decisive and are quick at making decisions) prefer a problem to be concise and well structured [15]. On the other hand, persons who score high in the perceptive scale are more concerned with seeing all sides of a problem and prefer flexibility.

Disposition on the extraversion scale also affects problem solving [15]. Individuals with high extraversion have greater tendencies to be sociable and require engagement with others. When given a problem, extraverts are more likely to discuss the problem in order to ascertain clarity and understanding, while introverts are more likely to take time and think about the problem before they begin. This implies that introverts would generally take longer before attempting a solution but are more likely to have a well-defined path toward finding that solution. Furthermore, introverts are more likely to take time to understand important concepts while extraverts require feedback on the correctness of their ideas.

While extraversion and judgment can be used as a predictor of a user's approach to a problem, similar tendencies can also be deduced from an individual's neuroticism orientation. Neuroticism measures a person's degree of emotional stability. Individuals who score high on the neuroticism scale are more prone to experiencing negative emotions such a stress and anxiety. As recent study by Uziel [16] has investigated the correlation of neuroticism and extraversion and suggest a negative correlation between them when affective states are considered. An individual's neuroticism disposition can also be a predictor of their 


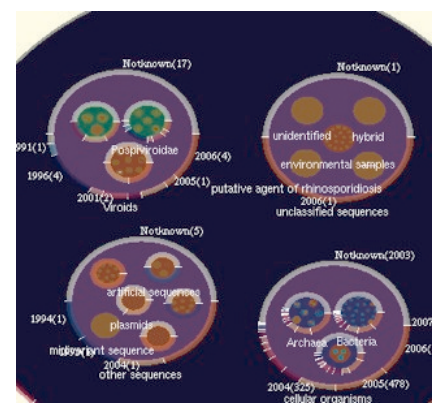

(a) GVis

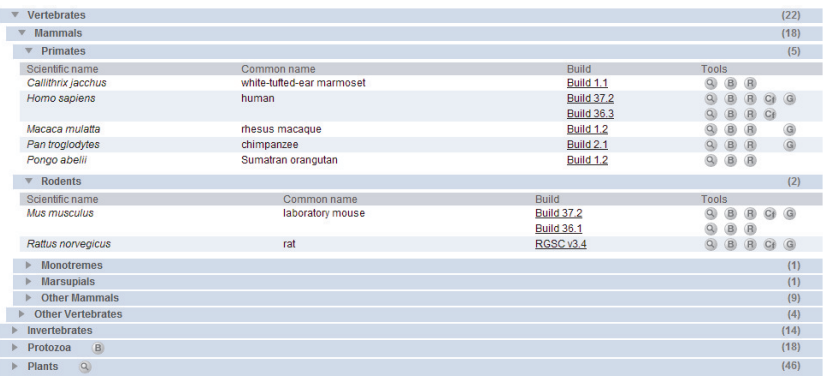

(b) NCBI Map Viewer

Fig. 2. The two interfaces used in Green et al.'s study [3] of personality differences in visual analytics use.

problem-solving approach [17]. Persons with high measures of neuroticism are more like to have lower perceived problem-solving skills and are less likely to make decisions when risks are involved. More neurotic people also take more time to solve problems, as was found by Farley [18], who found a curvilinear correlation between neuroticism and time spent solving a problem. Individuals who are measured on the neuroticism scale as average are significantly faster on problem-solving tasks than the combined low and high scoring people.

Several studies have also established correlations between locus of control and neuroticism [19], [20]. These traits are multidimensional constructs and are comprised of similar traits such as anxiety and selfesteem. It is therefore not surprising that locus of control may also affect problem solving ability and style. Of the three personality dimensions we study, locus of control has been found to have the most pronounced correlation to problem solving using visualizations.

\subsection{Locus of Control}

Much of the direct background for this work comes from Green et al.'s research [3], [4] on personality factors in visual analytics use. Their work found effects on interface performance from three psychometric measures: locus of control (LOC), neuroticism, and extraversion. Locus of control [21] measures the degree to which a person sees herself as in control of events (internal LOC) as opposed to seeing her fate as controlled by outside events (external LOC).
Locus of control has been associated with a number of significant practical outcomes. People with a more internal LOC tend to be more effective at work [22] and on many academic measures [23], and are more capable of coping with stress [24]. Locus of control in an academic setting is also associated with different learning styles. Cassidy and Eachus [25] found a positive correlation between external LOC beliefs and a tendency to use "surface learning" approaches, where the student attempts to fit the demands of an assignment instead of "deep learning," which focuses on the underlying principles being taught. On the other hand, students with an internal locus of control were more likely to use a deep learning approach. Such findings suggest the possibility that locus of control is related to how a user approaches problem-solving tasks in a novel setting, which may explain why it influences use of a novel visualization technique.

Green et al.'s work studied the effect of locus of control and other personality dimensions on both procedural and inferential learning in a GVis, a visual analytics interface (Figure 2(a)) versus NCBI Map Viewer, a more traditional web interface (Figure 2(b)). The procedural tasks they studied involved searching for a specific piece of information in a genomic database, while inferential tasks were those in which a user had to make a more open-ended comparison between two items. In both cases, the tasks were prompted by questions of the kind found in a typical usability study. The findings from both experiments suggested that the web table interface was more conducive to answering procedural questions and the findings from their first experiment suggest that the visual interface is more conducive to answering inferential questions.

In the first experiment, they found that participants with an external locus of control completed inferential tasks more quickly than those with an internal locus. This effect was more pronounced in GVis. In the second experiment, they studied only procedural tasks and found that, in contrast to inferential tasks, those with an internal locus of control completed procedural tasks more quickly. They also found correlations between the Big Five personality dimensions of neuroticism and extraversion and completion times for search tasks. More neurotic and more extraverted participants were significantly faster at finding the target of a search task in both interfaces.

In these studies, there were differences not only between interface structures but also between their interaction techniques. For example, GVis used a drilldown zooming technique while Map Viewer used a menu-driven interaction technique. In our work, we build upon these findings by seeking to isolate the factors of the interface structure that cause the different effects between those with an internal or external locus of control.

The amount of data we have about how different user types react to different interfaces is rapidly 
increasing. Our goal as visualization researchers is to make sense of this data within the context of models of the user. In order to apply this knowledge to improve design, we need to know not just what differences exist between users, but why. This work is an attempt to answer that question relative to Green et al.'s findings on personality and interface design.

\section{EXPERIMENT}

Research by Green et al. [3], [4] suggests that locus of control and other personality traits influence an individual's use of a complex visualization system. However, the reason for this relationship is not obvious from previous research. We propose that rather than an interaction between between locus of control and specific complex visualizations, the observed pattern may in fact be a correlation between locus of control and visual layout.

For example, consider the nested circles used in the GVis system from work by Green et al. [3], [4]; these structural elements are visually dominant due to their unusual shape and large size with respect to the surrounding textual elements. In contrast, Map Viewer uses a more subtle indentation-based structural expression which is dominated by text. Apart from using different visual encodings and interaction styles, these two designs represent significantly different visual layouts of the same underlying data. Exploring how those layouts differ may help explain why locus of control interacts significantly with them.

To more closely investigate this interaction, we conduct a comparable study in which the test visualizations are more tightly controlled. We restrict the variation between our four test interfaces to visual layout style, holding interaction metaphor and visual encoding consistent across all interfaces. We hypothesize that, even under this simplified setting, participants with a more internal LOC will have difficulty with layouts that depend on a strong containment metaphor, while participants with a more external LOC will show a greater willingness to adapt to a variety of visual layouts.

To test this hypothesis, we performed a user study in which participants were asked to answer search and inferential questions about data in four simple hierarchy visualizations (Figure 1). The four views were designed to express an increasingly visually explicit containment metaphor for the hierarchy, ranging from a list-like view that only used indentation to show hierarchical structure to a view that used large nested rectangles. Like Green et al., we measured personality traits of the participants beforehand in order to test whether Locus of Control, Neuroticism, and Extraversion affect a participant's ability to use these visualizations.

Green et al. [3] used two real-world systems in their work, which has the benefit of providing a realistic testing environment. However, this also makes it difficult to isolate exactly which aspects of the two designs prompted the differing user behavior they found. As the long-term goal of this work is to assist designers in choosing how to display information for varying user types, knowing exactly which elements of the design should be altered is vitally important. Therefore, our intention in designing the visualizations used in our study was, as much as possible, to isolate the factor of layout style which we hypothesized to be the key to these differences.

Before viewing the data, each participant was given a personality test, including the Locus of Control Inventory [21] and the Neuroticism and Extraversion scales of the Big Five Personality Inventory [26]. The participant was then presented with a series of tasks to perform on each visualization. The order in which the visualizations were presented was randomized, and the user's ability to successfully complete tasks using each visualization was recorded.

\subsection{Participants}

We recruited 240 participants over Amazon's Mechanical Turk service. Altogether, it took approximately two days to collect data from all participants. Mechanical Turk is an online job market in which people can be recruited for brief tasks and paid for their efforts. This service has become increasingly popular for use in online experiments, as a large number of relatively diverse participants can be processed very quickly [27]. Reservations remain about using Mechanical Turk among the human-computer interaction and visualization communities. However, since Mechanical Turk helps correct for a number of the traditional limitations of online studies, such as the possibility of vote flooding and the lack of incentive for completion [28], it is gradually becoming more accepted as a user study platform. It can be particularly useful in studies such as this, in which there is a ground truth by which to measure results [29] and the possibility of incentivizing accurate responses through bonuses [28].

That said, some limitations remain with interpreting online studies in general. Chief among these is environmental control. In an online study, it is impossible to know whether a participant's environment is noisy or distracting, or whether the participant is doing something else while performing the study. While these limitations should be kept in mind, this is a study with a clear ground truth and a task that should not be strongly affected by varying computing setups. In addition, our task questions were designed not to be answerable using a search engine. Under these circumstances, we argue that the advantages of using Mechanical Turk to obtain a high number of users outweigh the potential issues.

Of the 240 participants, four did not report their age or gender. Of the rest, there were 124 males and 112 
females. Self-reported age ranged from 18 to 62, with a mean of $26.7(\sigma=9.5)$. Our participants reported an average locus of control score of $3.61(\sigma=.59)$. This is slightly lower than scores reported in other publications that use this particular scale [30], [31]. For example, Lapierre and Allen [31] find a mean locus of control of $4.03(\sigma=.61)$ for a participant pool of 205 employees in various professions. This difference may reflect the broader demographics of Mechanical Turk workers versus participants in traditional psychological studies. Locus of control in particular is often studied in the context of work or education, meaning the participants in these studies may have different educational or economic backgrounds than the general population. However, as we did not collect such demographics from our participants, we can only speculate on this point.

\subsection{Materials}

Participants were initially given two questionnaires to measure the aspects of their personality which are relevant to our hypotheses: a scale to measure the Big Five personality dimensions of Extraversion and Neuroticism and a locus of control scale to measure the degree to which they see themselves as in control of or controlled by external events. Both scales were taken from the International Personality Inventory Pool [26] and were combined to form a 40-question survey. Neuroticism and Extraversion were included for comparison with Green et al.'s results, although they are not the focus of the current analysis.

Green et al.'s study used two fully functional data exploration systems with many differences between them. For our study, we wished to isolate as much as possible the variable of layout style, so we created a set of four very specific visualizations (Figure 1). The first of these, V1 (Figure 1(a)) displays a tree in a simplified Windows Explorer style, using only indentation to indicate hierarchical relationships. This is representationally similar to the webpage organization used by Map Viewer in Green et al.'s work. The fourth view, V4 (Figure 1(d)) uses a nested boxes display that relies heavily on the visual metaphor of hierarchy as containment [32]. Although it uses rectangles rather than circles and a very different interaction style, this view is representationally similar to the nested bubbles of the GVis visual analytics system.

Between these two extremes, we designed two intermediate views. V2 (Figure 1(b)) is very similar to the indentation style of $\mathrm{V} 1$, but adds borders around the tree nodes to suggest a containment metaphor. V3 (Figure 1(c)) breaks the strictly vertical layout style used in V1 in favor of a horizontal layout closer to that used in V4, but still employs indentation to organize the levels of the hierarchy. These views are intended to provide cases that interpolate between the two layout styles used in Green et al. Our use of such simplified views may raise the concern that our results do not directly apply to more realistic visual analytics scenarios. However, since we are partially attempting to replicate results from a study that employed realworld analysis systems, we feel this approach is complementary to previous work. If our results are similar to those found by Green et al, it would demonstrate that this simplification still maintains the important differences between the systems used in their study.

Furthermore, if a trend can be found to increase from $\mathrm{V} 1$ to $\mathrm{V} 4$, it would show that our intermediate views do indeed capture the major differences between the two views. Since the intermediate views primarily differ in the degree to which they express either a list-like or containment metaphor, this could support our argument that the finding is largely based on the different user groups' willingness to adapt to one metaphor over the other. That said, layout is a complex factor that by nature is made up of many dimensions. While this study design is intended to keep these layout differences as controlled as possible, there are like other aspects of the layout, such as dataink ratio or size of visual elements, which cannot be entirely ruled out as factors. Nonetheless, this study design can at least test whether layout factors in general can lead to a locus of control effect without differences in visual encoding or interaction.

Apart from these specific design differences, we endeavored to maintain consistency between the four views whenever possible. They all use the same font size and folder icons. Each visualization also has the same interaction style, based on the collapsing folders metaphor seen in a standard desktop file system. This may somewhat bias the results in favor of V1, which most closely resembles the interfaces used in file systems. Nonetheless, we argue that maintaining interaction consistency is important enough for isolating design factors that this is worth the tradeoff.

In addition, we implemented the restriction that only one subtree could be open at one time. If a user expanded one branch of the hierarchy and then attempted to expand a node in an unconnected branch, the first branch would automatically collapse. This was intended to keep the amount of potentially visible information consistent across the four views. Having several subtrees open is fairly easy in V1, which is purely vertical, but it would be difficult or impossible to open an infinite number of subtrees in V4 without making the lower-level nodes too small to display an entire label. Finally, to see genome data about individual species, the participant hovered the mouse over the species name to bring up a tooltip. Our goal with this interaction style was to keep the four views as consistent as possible except in how they visually organize the space.

The datasets presented in these four visualizations were four subsets of the full taxonomic tree from the National Center for Biotechnology Information's 
TABLE 1

The eight task questions seen in the study.

\begin{tabular}{|c|c|c|}
\hline Dataset & Question Type & Question \\
\hline Amphibia & $\begin{array}{l}\text { search } \\
\text { inferential }\end{array}$ & $\begin{array}{l}\text { Within the classification "Batrachuperus," which species was most recently updated? } \\
\text { Under "Anura," find the classification "Bufo" and note the subclasses it contains. There is another classification under } \\
\text { "Mesobatrachia" that has something notable in common with "Bufo." Find that classification. }\end{array}$ \\
\hline Aves & $\begin{array}{l}\text { search } \\
\text { inferential }\end{array}$ & $\begin{array}{l}\text { Under the classification "Falco," find the species with a "Length" value over } 18000 . \\
\text { Looking in "Sphenisciformes," find the classification "Eudyptula" and note the species under it. Now look in } \\
\text { "Threskiornithidae" for a classification that has something notable in common with "Eudyptula." }\end{array}$ \\
\hline Eutheria & $\begin{array}{l}\text { search } \\
\text { inferential }\end{array}$ & $\begin{array}{l}\text { Within the classification "Tarsius," find the species which was most recently updated. } \\
\text { Under "Caniformia," find the classification "Canis" and note the subclasses and species it contains. Now find another } \\
\text { classification under "Ursidae" that has something notable in common with "Canis." }\end{array}$ \\
\hline Lepidosauria & $\begin{array}{l}\text { search } \\
\text { inferential }\end{array}$ & $\begin{array}{l}\text { Under the classification "Bipes," find the species with the lowest "Length" value. } \\
\text { Within "Scincomorpha," find the classification "Lacerta" and note the species under it. Now look in "Crotalinae" for } \\
\text { a classification which has something in common with "Lacerta." }\end{array}$ \\
\hline
\end{tabular}

Genome database [33]. Each dataset represents a phylogenetic tree where leaf nodes are individual species. At the leaf level, there is data on the genome mapping data available for that species, such as the date the entry was updated and the number of proteins and genes in the database. This is similar to the data used in Green et al., but does not include all the information found in the Map Viewer subset of the database. We chose to show less data at the leaf level in order to present more data overall and more complex trees. The four datasets had, on average, 98.75 leaf nodes (i.e. individual species) and 114.75 non-leaf nodes in the phylogenetic tree. There was some variety in the branching factors and overall structure of the trees, although this was not carefully controlled for. However, since the datasets were ultimately balanced with respect to the view types, these differences should not significantly affect our results.

We considered the unfamiliarity of the datasets to be beneficial to our study, since we could trust that participants would need to consult the views in order to answer the task questions we presented them. Following Green et al. [4], these questions were divided into search tasks and inferential tasks. In both cases, tasks took the form of questions that participants were expected to consult the visualization to answer. This is similar to the methodology used in most visualization evaluation studies. These two question types are meant to represent simple data lookup and more complex analytical tasks, although they are simplified versions of the real-world versions of these tasks. We expect to see more differences in the inferential questions, since these are more likely to require understanding of the structure of a dataset rather than simple navigation ability.

The search questions asked the participants to find a single species within a classification that had a certain property. For example, "Within the classification 'Batrachuperus,' which species was most recently updated?" The verbal metaphor for tree structure used in these questions was varied between a levels metaphor and containment metaphor, to avoid any potential confound of metaphor compatibility [32]. Participants were asked to write the name of the species they found within a text field. The inferential questions were more open-ended, asking the participant to find a certain classification, then find another classification in another part of the taxonomy that had something in common with the first. For example:

Under "Anura," find the classification "Bufo" and note the subclasses it contains. There is another classification under "Mesobatrachi" that has something notable in common with "Bufo." Find that classification.

This was usually a similar pattern of names or numbers of species that fell under the classification. For example, the classification "Bufo" contained a classification also called "Bufo," and the correct answer was another classification that contained a child node with the same name as itself. Since correctness may be harder to judge in questions with free text responses, participants in this case chose their response from a list of four multiple choice answers (as well as a "None of the Above" response). Each dataset was associated with two task questions: one search question and one inferential question. Participants therefore saw eight task questions altogether (Table 1).

\subsection{Procedure}

After selecting the study task from the Mechanical Turk website participants were asked to fill out a 40question personality scale by rating each item from 1 (strongly disagree) to 5 (strongly agree). Once they were done, they read instructions for the remainder of the experiment.

The main portion of the experiment consisted of four sessions, one with each of the four views. The sequence in which these views were presented was counterbalanced to prevent ordering effects. Four datasets were randomly distributed between the four views, such that each dataset was seen exactly once.

When they first saw the visualization, participants were asked to familiarize themselves with the in- 
terface for as long as they liked. When they were done, they clicked on a button labeled "Start" and were presented with the search question. They again had unlimited time to interact with the visualization and find the answer. When they were satisfied, they clicked on a "Ready to Answer" button and were presented with a text field to fill in their answer. Interaction with the visualization was then locked to limit the recorded response time to the actual interaction time and ignore the time used to type in the answer.

After answering the search question, the participant was presented with an inferential question on the same dataset. The procedure for the inferential question was the same as for the search question, except that participants were presented with a set of multiple choice responses instead of a text field to record their answer. The responses were presented in random order and were not visible during the interaction period. Once both questions were answered, participants were shown a brief four-question preference survey on how much they liked the visualization. The questions in this survey were:

- This system was easy to use.

- The way this system arranged information made sense to me.

- I understand the data better after using this system.

- I enjoyed using this system.

Participants rated how much they agreed with each of these statements on a five-point scale. After completing all four sessions, participants were asked to provide their age, gender, and any comments about the study in a form on the Mechanical Turk site.

Overall, the experiment lasted approximately one hour. We measured the time spent in the initial training period, the time taken in the interaction period, and the time needed to record their response, as well as whether their response was correct or incorrect. In addition, we calculated each participant's locus of control, neuroticism, and extraversion based on their personality scale responses, and averaged their preference survey responses to generate a Preference Score for each visualization.

\section{Results}

The task questions proved quite challenging, with an overall accuracy of $68.6 \%$ correct responses on search tasks and $47.1 \%$ on inferential tasks. This, coupled with the large amount of time spent interacting with the views, should be kept in mind when interpreting the following results. Across all participants and question types, no view condition was more or less difficult in terms of accuracy $\left(\chi^{2}(3, N=1919)=3.7\right.$, $p=.29)$ or correct response time $(F(3,1109)=.57$, $p=.63)$. As our primary interest is the influence of a participant's personality factors, further analysis focuses on participants grouped by personality type. We found support for our hypothesis that participants

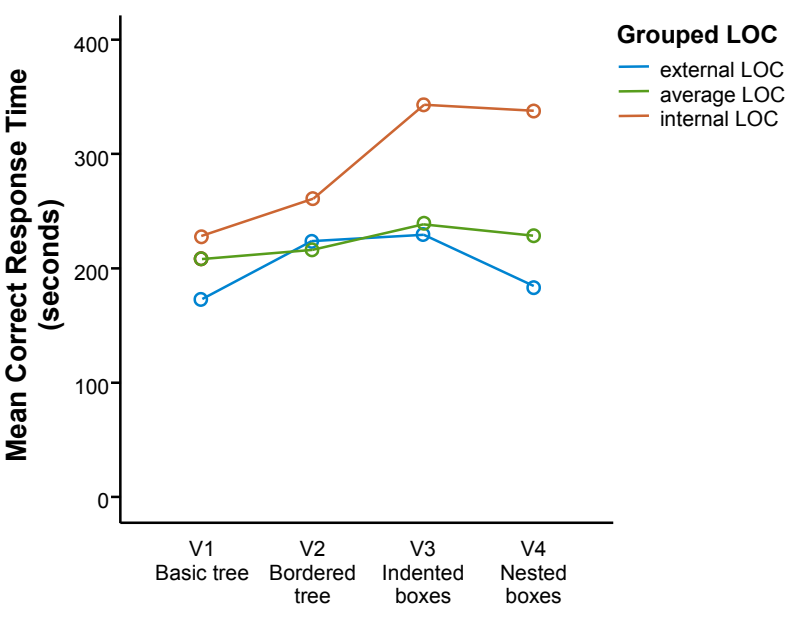

Fig. 3. Inferential task response time for correct answers only across the four view conditions and three locus of control (LOC) groups. Participants with a highly internal LOC, who see themselves as in control of external events, were much slower than other participants at answering inferential questions in V4, a visualization that uses a strong nested-boxes visual metaphor. Participants with a highly external LOC, who see themselves as controlled by outside events, are relatively more likely to perform quickly on V4.

with a more internal locus of control would have more difficulty with views similar to V4. While we found that participants with a more external locus of control did perform very well with V4, we did not find a corresponding trend in which their performed decreased on views similar to V1. We found partial support for Green et al.'s finding that more neurotic participants would perform better with the more explicitly structured views, but no support for the their finding that less extraverted participants would perform better with these views.

\subsection{Effects of Locus of Control}

We initially divided participants into three groups based on their score on the Locus of Control scale. Participants with a score lower than one standard deviation from the mean (i.e. less than 3.01) were classified as external LOC users. Those with a score greater than one standard deviation from the mean (i.e. greater than 4.21) were classified as internal LOC users. The rest were classified as average LOC users.

The independent variables we analyzed to test our hypothesis were Question Type (search task versus inferential task), View Condition (V1, V2, V3, or V4), and LOC Group (external, average, or internal). Our overall model was therefore a $2 \times 4 \times 3$ Univariate Analysis of Variance (ANOVA). This overall model was significant for the variable of Correct Response Time $\left(F(23,1109)=10.67, p<.01, \eta_{p}^{2}=.18\right)$. Correct 


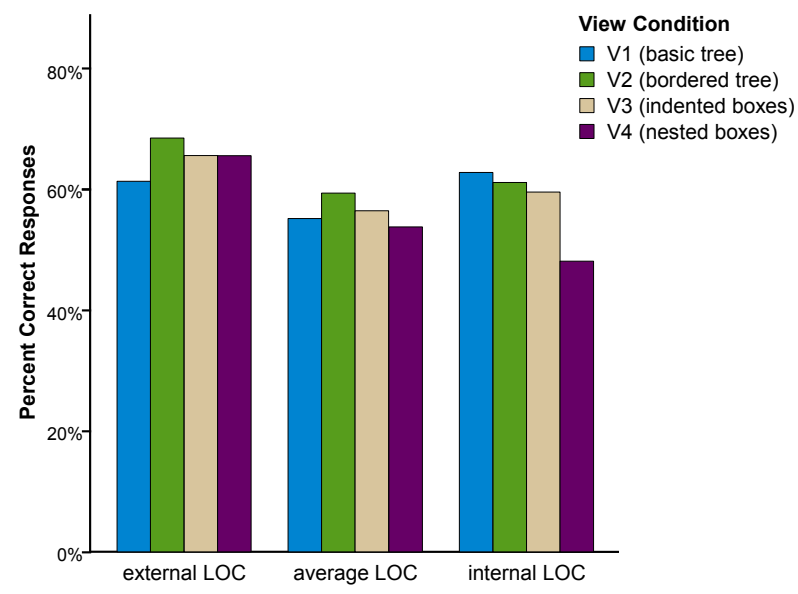

Fig. 4. Percentage of correct answers in both question types across the four view conditions for participants grouped by their locus of control score. Participants with a more external locus of control were more accurate overall, while the other groups performed poorly with V4.

Response Time only included correct responses, and represents only the time spent interacting with the view, not the time spent writing or choosing a response.

In addition, there were significant main effects of question type $\left(F(1,1109)=158.86, p<.01, \eta_{p}^{2}=.13\right)$ and locus of control $(F(2,1109)=5.38, p<.01$, $\left.\eta_{p}^{2}=.01\right)$. Unsurprisingly, search questions were answered faster than the more difficult inferential questions. Overall, internal LOC participants were slower at answering questions correctly $(M=183.4$ seconds, $S D=11.6$ ) than external LOC participants $(M=147.9$ seconds, $S D=10.3)$. There was also a significant interaction between view condition and question type $\left(F(3,1109)=3.01, p<.05, \eta_{p}^{2}=\right.$ $.01)$ and between question type and LOC group $\left(F(2,1086)=5.93, p<.01, \eta_{p}^{2}=.01\right)$. The reason for the latter is that internal LOC participants are much slower than the others on inferential tasks, but are the same speed when answering search tasks. The source of the interaction between view and question type is that, while inferential questions were answered more slowly than search questions in all views, this difference was significantly larger in V3. For inferential questions, V3 produced the slowest response times of all views, but produced the fastest for search tasks. We focus our subsequent analysis on inferential questions, since it was on these questions that the individual differences between participants were most substantial.

The results relevant to our main hypothesis are summarized in Figure 3, which shows inferential question response times across view types and participants grouped by locus of control. We found that participants with an external LOC answer inferential

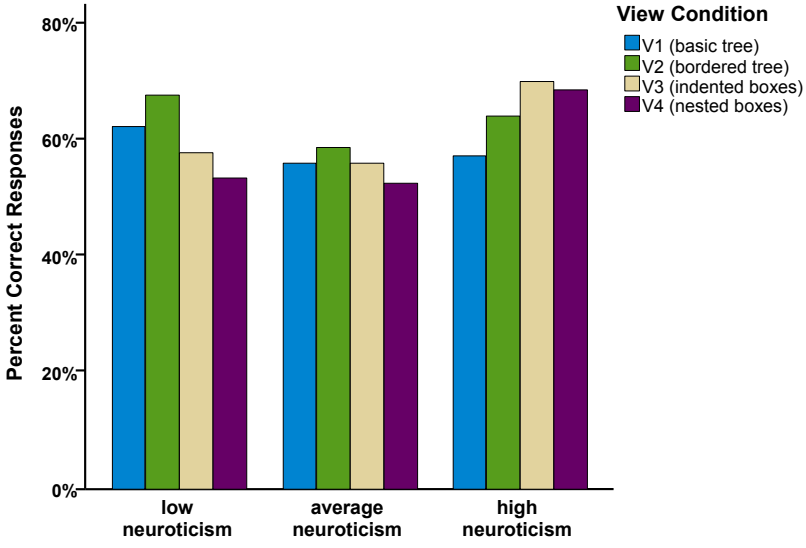

Fig. 5. Percentage of correct answers in both question types across the four view conditions for participants grouped by their neuroticism score. More neurotic participants performed more accurately with visualizations that used a more container-like layout, while participants in other groups displayed the opposite trend.

questions in V4 (the nested boxes) faster than other participants, although they answer questions in V1 faster as well. Internal LOC participants show a clear trend of slower performance from V1 to V4, although there is no distinction for these participants between V3 and V4. Average LOC participants show no response time difference between the four views. We also found that raw locus of control score correlates significantly with correct response time on nested boxes but no other condition $(r(104)=.23, p<.05)$. For search tasks, there was no significant response time difference for any participants between the four views.

To test overall accuracy, we used a Pearson's chisquare test on correctness (in both question types) and grouped locus of control. Participants with an external LOC answered more questions correctly overall $\left(\chi^{2}(2, N=1919)=7.7, p<.05\right)$, possibly due to their better performance on conditions V3 and V4. These results are summarized in Figure 4.

\subsection{Results by Big Five Personality Factors}

As with Locus of Control, we divided participants into high, low, and average groups for both Extraversion and Neuroticism using one standard deviation from the mean as dividing points. This split participants into three groups based on Extraversion: introverted (less than 2.29), average extraversion, and extraverted (greater than 3.86). For Neuroticism, these groups were low neuroticism (less than 2.02), average neuroticism, and high neuroticism (greater than 3.53).

We found no overall effects of either of the two Big Five measures on correct response time. As with LOC, we used a Pearson's chi-square test on correctness and grouped personality type to test differences in 
accuracy. Introverts were more accurate across all four views and both question types than extraverts $\left(\chi^{2}(2, N=1919)=34.15, p<.001\right)$. A similar effect was found for participants with high Neuroticism scores $\left(\chi^{2}(2, N=1919)=9.24, p=.01\right)$.

In addition, highly neurotic participants were significantly more accurate than the other groups in the high-structure V4 condition $\left(\chi^{2}(2, N=480)=6.12\right.$, $p<.05)$. In general, the more neurotic participants seemed to answer a higher percentage of questions accurately as views became more container-like, while the other groups showed the opposite trend (Figure 5). There was no equivalent significant effect for extraversion. Additionally, unlike Green and Fisher [3], we found no effect of either extraversion or neuroticism on response time for search tasks.

\subsection{Other Findings}

In addition to the personality variables that we measured, we analyzed our results based on the demographics and preference information we collected from participants. According to a repeated measures ANOVA on the preference scores for each visualization, V1 and V2 were rated significantly more positively than V3 and V4 $(F(3)=15.09, p<.001)$. These preference ratings displayed no significant correlation with accuracy or response time. There was also no relationship between locus of control and preference ratings for any of the four views.

Female participants answered more questions correctly than male participants $(t(231)=-2.31, p<$ $.05)$. We found that the age of participants correlated positively with overall response time $(r(1893)=.13$, $p<.001)$, so that older participants took more time interacting with the interfaces. Age had no effect, however, on accuracy, and did not correlate with any of the personality measures. In the following sections, we will discuss these implications of these findings for our hypothesis and for visualization design in general.

\section{Discussion}

Through this study, we demonstrated previously reported effects of locus of control in a simplified design that isolates layout style. This supports our main hypothesis that the way visual elements are spatially arranged is a significant factor of design that interacts with locus of control. This clarifies the earlier findings and suggests an explanation for this effect rooted in the relationship between locus of control and use of external representations.

\subsection{Replication and Expansion of Previous Find- ings on Locus of Control}

Our results replicate those of Green et al. [3], [4] in some cases, but not all. Error rates across the two experiments are not directly comparable. In the previous study, participants were allowed as many attempts as needed to answer a question correctly, with each mistake recorded as an error. However, both studies found that participants made more errors overall in the containment-metaphor visualization than in the more list-like view (V1 in this study, Map Viewer in [3], [4]). More significantly, external LOC participants in both studies responded faster to inferential questions than internal LOC participants, particularly in a visualization with a containment-based visual metaphor (V4 in this study, GVis in [3], [4]).

Together, these findings provide evidence that the effects of locus of control on visualization use can be replicated. Users with either an internal or external locus of control show performance differences in general on data exploration tasks, and additionally, each group performs better with different visualization styles. This suggests that locus of control may be a variable that merits further study, and that personality differences in general may be a valuable area for research in visualization.

We did not replicate previous findings on search task response times. Green et al. found that internal LOC participants responded faster to search tasks in GVis, and that neurotic and extraverted participants responded faster to search tasks overall. However, completion times for these tasks included incorrect responses and guesses made by participants, so our response times are not directly comparable for these questions. This was not the case for the inferential questions, where they recorded only the time to make a single response.

We speculate that the inferential questions forced the users to consider the structure of the data to a greater degree. The search questions may have simply measured a participant's ability to navigate the interface quickly, while the inferential questions asked them to characterize parts of the data in an openended fashion. Participants may have interpreted these questions in a variety of ways, allowing the structural elements of the visualization design to play a greater role in their thought process.

Although external LOC participants were faster than other participant groups at answering questions in V4, they were equally fast in V1. This finding does not fit our original hypothesis that internal and external participants prefer different types of visual layouts. It may be that the high familiarity of tree menus like V1 created a training effect that caused a break in the overall pattern. However, given the data, we cannot conclude that external participants perform better with containment views than with list-like views. Rather than a clear trend of group preference, a better interpretation of our results may be that external LOC participants are generally better able to answer inferential questions using unusual visualization layouts. An experiment controlling the potential confound of a highly familiar view is needed 
to test which interpretation is better supported.

Familiarity may also explain the higher preference scores across all participants for V1 and V2, though it is interesting that there was no correspondence between preference and performance. It is also possible that this lacking relationship may reflect the fact that participants were paid a bonus for correct responses, and therefore had an incentive to perform well despite disliking the interface. In any case, people may have felt that V3 and V4 were especially confusing due to their unusual appearance, but they were just as capable of answering questions with these interfaces.

\subsection{Findings on Other Personality Factors}

Compared to Green and Fisher [3], we found fewer notable differences between participants categorized by neuroticism or extraversion. In fact, these personality dimensions had no significant effect on response time, which is where the most dramatic effects of locus of control were found. On the other hand, we did find that these dimensions influenced participants' accuracy on search tasks.

In the case of neuroticism, our results provide support for Green and Fisher's finding that more neurotic participants generally perform better on search tasks with visual interfaces. The highly neurotic participants were significantly more accurate overall, aggregating all four views and both question types. As suggested by Green and Fisher, this may be explained by the theory that people with more neurotic or traitanxious personalities tend to be more attentive to problem-solving tasks up to a certain level of complexity [34].

However, we also found that this effect was especially pronounced in the case of V3 and V4, the most container-like of the four views. Notably, the low-neuroticism participants performed more poorly on these two views than on V1 and V2, which likely contributes to the overall relationship between neuroticism and accuracy (Figure 5). This mirrors the findings on locus of control (see Figures 4 and 5) and suggests that less neurotic participants, like the more internal ones, are less able to make sense of these types of visual layouts. It is possible that the greater attentiveness of these users makes it easier for them to learn an unfamiliar interface. Lower neuroticism also correlates with a more internal locus of control, and it is possible that the two scales measure similar qualities that both indicate different aspects of a user's unwillingness or inability to adapt to unusual external representations. An alternate explanation is that the neurotic participants may put more pressure on themselves to complete a question correctly rather than abandoning a task due to an unfamiliar visualization.

In the case of extraversion, our results seemingly diverge from Green and Fisher's. They found that more extraverted participants responded more quickly to search tasks, and we found that more introverted participants were more accurate on all task types. One possibility is that, under some circumstances, extraverted users respond more quickly but less accurately. That said, we did not find any significant results regarding response time in the current work, so this remains speculative. However, this hypothesis is supported by previous work on introversion and problem solving which finds that more introverted people tend to take more time to think through problems [15]. This extra time may have been particularly helpful in a situation where they had to reason with unfamiliar visual interfaces and datasets.

Since extraversion showed no significant relationship with view type, the overall profile of a participant in our study who performed well with the more containment-like visual layouts is someone who had an external or average locus of control and was highly neurotic. As neuroticism measures emotional stability, and locus of control the degree to which a participant feels in command of situations, these findings may indicate that feeling somewhat out of control can be an advantage when it comes to making sense of novel visualization designs. It is worth noting that both of these dimensions tend to be negatively correlated with job performance and other practical outcomes [22]. Taken together, this suggests the possibility that persons who struggle with more standard tasks are better suited to thinking with complex visual representations. This possibility warrants further investigation. At the same time, a novel visualization design, while helpful for some users, may be a hindrance to users who already perform a task well using their own methods.

Further work is needed to understand these patterns of users who perform better or more poorly as visual layouts tend towards a strong containment metaphor. It is necessary to examine whether these findings can be generalized beyond this specific task and data type as well as whether they can be generalized to different sets of visual metaphors. Nonetheless, we argue that our findings, particularly those on locus of control, serve as a step towards a better understanding of the externalization theory of visualization and how it works for different types of users.

\subsection{Locus of Control and External Representa- tions}

By isolating the variable of layout style in this study, we have shown that simplified layout changes can produce locus of control effects similar to those found in a user study that compares real-world systems. This provides evidence that layout is a key factor in mediating this effect, independent of the effects of interaction style, visual encoding, and general differences between traditional interfaces and visual analytic systems. Although the current work does 
not directly support a causal explanation, we can speculate on why the different layouts we designed interacted with participant locus of control the way they did. We argue that the explanation for the behavior we observed is that participants with an external locus of control are willing to adapt more readily to visualization styles that employ a strong structural metaphor.

The four views in our study range from one (V1) which is dominated by white space and uses subtle visual organization to one (V4) which is dominated by screen elements expressing a visual metaphor of nested boxes. We argue that these views represent a progression from visually implicit hierarchical structure to structure made visually explicit through layout and the use of lines and fills. V1 (Figure 1(a)) uses only a single dimension of spatial organization (horizontal indentation) and makes little distinction between leaf nodes and parent nodes. These factors make the hierarchical structure of the data less explicit. For example, two sibling nodes (such as Rhinocerotidae and Equidae in Figure 1(a)) can be visually separated to the point that their relationship is not immediately obvious. Because of this, we argue that V1 is the least structurally dominant of the four views.

After V1, each visualization in the sequence adds at least one visual element used to draw attention to the hierarchical structure of the data. The borders in V2 (Figure 1(b)) slightly emphasize the nesting of child nodes within parent nodes; for example, Rhinoceros and its two children are clearly grouped. V3 (Figure 1(c)) takes this further by highlighting the names of parent nodes and arranging child nodes horizontally to emphasize a spatial metaphor of containment. Finally, V4 (Figure 1(d)) gives the parent node a strong visual emphasis with centering and a title bar and collects the child nodes together at the top of the parent to make sibling relationships obvious. This is the least "list-like" of the four views and uses the most "ink" and screen space to express hierarchical relationships.

The connection between this use of explicit visual structure and locus of control may be explained by the body of thought that views visualization as an external mental representation. The field of distributed cognition [35] sees mental processes such as problem solving and memory as relying not only on knowledge stored in the mind, but also on knowledge stored in a person's environment, in the form of physical objects, information artifacts, and other people. Applying this perspective to visualization, Liu et al. [36] have argued that a primary benefit of visualization is the externalization of information. Externalization makes problem-solving more efficient and accurate by substituting quicker perceptual processes for cognitive processing of information. Furthermore, studies have shown that the form of these representations can influence problem-solving strategies.
Users with an external locus of control are those who expect the outside world to dominate their fate. Taking a distributed cognition approach, it is possible that these users also rely more heavily on outside representations, rather than internal mental representations, when solving problems or making sense of information. Conversely, those with an internal locus of control may prefer to perform cognitive tasks more internally, relying less on external representations. In general, this makes people with an internal locus of control more adept at problem-solving and learning, as the extensive literature on locus of control has shown. The results of this study may be surprising in light of these findings. One possible explanation is that the same tendency of internals to rely on internal representations may make it more difficult to use complex external representations found in a visual analytics system. It is also possible that this performance difference is only seen as individuals with an internal locus of control first interact with an unfamiliar system. We hypothesize that their performance may dramatically improve as they transition from novices to experts of the system. This explanation lends support to previous findings of performance achievements by internals; however this is mere speculation and requires further study.

If this is indeed the case, a visualization with a highly explicit and unfamiliar visual structure may be more jarring for an internal LOC user. Someone with an external locus of control may be more willing by nature to adapt her thinking to the external representation, while the user with an internal locus may be going through a more difficult process of fitting the external representation to her own ideas of what the data is like. Cassidy and Eachus's work [25], discussed in Section 2.3, implies that the "surface learning" approach taken by external LOC students is academically harmful, and based on well-established findings in locus of control research, this does seem to be the case in general. However, this very tendency to focus on surface structure may be beneficial in the context of learning a new visualization system. Our external LOC participants were just as fast with a novel visualization as they were with the kind of indented list they see on their computer desktop every day. There may be other ways of interpreting these effects, and for now, this is a hypothesis for future research, not a firm conclusion. Nevertheless, this potential ability to make an advantage out of a personality style that is usually considered problematic suggests intriguing future directions in the application of visualization to learning.

Taken together with previous work, these findings contribute to the case for an externalization-based view of how people perform complex tasks with a visualization. Furthermore, they imply that this externalization process varies greatly between people and situations, which may be a significant factor in the 
difficulty of controlling and interpreting evaluations of visualization systems. This increased understanding of how externalization relates to individual differences not only contributes to visualization theory, but also has practical implications for the design of visualization systems.

\section{IMPLICATIONS FOR DESIGN}

These studies and others like them provide mounting evidence that personality and design style can have a significant effect on whether a user accepts a visualization design. It is possible that a user's personality can serve as shorthand for subtle cognitive style differences that are not easily measurable otherwise, but which gain importance in the exploratory context of visualization use. The significant effect of locus of control on performance suggests that it measures something particularly important to visualization use, and so in this section we focus on how understanding locus of control can be used to improve visualization design.

When we give users a novel visualization, we are essentially asking them to give up some control over their thinking processes. Some users will find this helpful, while others may find it a hindrance. We argue that a visualization designer should have a sense of how willing a given user will be to take on an external representation, and know how to design a visualization that makes it more or less difficult to ignore the structural aspects of that representation.

Based on our findings, a useful guideline for adaptation would be to increase the amount of explicit structure for users that might have a more external locus of control. Users with a very internal locus of control will likely perform best with a visualization style that uses simple spatial organization and minimal borders, outlines, and other grouping elements. In practice, this type of design may correspond to the maximized "data-ink ratio" argued for by Tufte [37]. External LOC users, on the other hand, may perform more efficiently with a visualization style that violates this classic guideline by including more nonfunctional elements such as borders, fills, and outlines to call attention to a specific information structure. In addition, this type of user may have an easier time working with visualizations that use a twodimensional spatial layout to organize information.

Although it would be impractical to directly measure a user's locus of control and adapt the visualization accordingly, future work can address the possibility of using this principle to guide design. Our research views locus of control as predicting the degree to which a user will, by innate disposition, prefer her own internal mental models (internal LOC) versus being willing to adapt to an external representation (external LOC). This general principle, then, may apply in other situations where users are likely to prefer a pre-existing mental model or problem-solving process for reasons other than personality. Expert users, for example, may be more resistant to visualizations with highly explicit structure. Likewise, a user group with a highly standardized analysis process should be given visualizations with low structural emphasis. A user group which is likely to approach a problem in a more exploratory mode may find it easier to work with visualizations that makes the structural organization of data explicit.

While an analysis of our results suggests possibilities for design, more research is needed to formalize the growing body of insights into individual users and visualization. Future work in this domain should include the development of formal design guidelines driven by a more comprehensive analysis of individual differences and their relationships to visual design elements.

\section{Conclusion}

In subsequent work, we hope to combine these findings with other work in individual differences to pursue more complete models of the visualization user. As Yi [38] has argued, major advances in understanding these individual differences must come from collecting knowledge across studies. This work is one attempt at doing so, and much more can be done to find commonalities among the measures and findings on how users adapt to visualizations.

In this paper, we have contributed findings on how users with different personality types react to varying layout styles used in a hierarchy visualization. We found evidence that systematic differences in layout style can indeed influence a user's response time and accuracy with different types of visualizations that are informationally equivalent but differ in layout. These findings seem to fit a pattern in which users with a more external locus of control are more efficient at using a visualization which uses a highly explicit visual metaphor than users with a more internal locus of control. We expect that these findings can serve as a step towards better understanding of why subtle differences between users' personality styles can have a surprising influence on visualization use.

\section{ACKNOWLEDGMENTS}

The authors wish to thank Tera M. Green and Brian Fisher for their helpful discussions and collaboration. This material is based in part upon work supported by the International Program of the Department of Homeland Security under grant number 2009-ST-108000007. Any opinions, findings, and conclusions or recommendations expressed in this material are those of the authors and do not necessarily reflect the views of the Department of Homeland Security. This material is based in part upon work supported by the National Science Foundation under Grant No. 
CIF-B-195. Any opinions, findings, and conclusions or recommendations expressed in this material are those of the authors and do not necessarily reflect the views of the National Science Foundation.

\section{REFERENCES}

[1] S. E. Palmer, Vision science: Photons to phenomenology. MIT Press., 1999, vol. 1.

[2] C. G. Healey, "Perception in visualization," North Carolina State University, http://www.csc.ncsu.edu/faculty/healey/PP, Retrieved March 2011.

[3] T. M. Green and B. Fisher, "Towards the personal equation of interaction: The impact of personality factors on visual analytics interface interaction," in IEEE Visual Analytics Science and Technology (VAST), 2010.

[4] T. M. Green, D. H. Jeong, and B. Fisher, "Using personality factors to predict interface learning performance," in Hawaii International Conference on System Sciences 43, January 2010.

[5] A. Dillon and C. Watson, "User analysis in HCI: The historical lesson from individual differences research," International Journal of Human-Computer Studies, vol. 45, pp. 619-637, 1996.

[6] C. Conati and H. Maclaren, "Exploring the role of individual differences in information visualization," in Advanced Visual Interfaces. ACM Press, 2008. [Online]. Available: http://www.cs.ubc.ca/ conati/my-papers/AVI2008-cameraWithInteraction.pdf

[7] B. Allen, "Individual differences and the conundrums of usercentered design: Two experiments," Journal of the American Society for Information Science, vol. 51, no. 6, pp. 508-520, 2000. [Online]. Available: http://www3.interscience.wiley.com/cgibin/fulltext/71001471/PDFSTART

[8] C. Chen, "Individual differences in a spatial-semantic virtual environment," Journal of the American Society for Information Science, vol. 51, no. 6, pp. 529-542, 2000. [Online]. Available: http://www3.interscience.wiley.com/cgibin/fulltext/71001476/PDFSTART

[9] C. Ziemkiewicz and R. Kosara, "Preconceptions and individual differences in understanding visual metaphors," Computer Graphics Forum, vol. 28, no. 3, pp. 911-918, 2009, proceedings EuroVis.

[10] L. R. Goldberg, "An alternative "description of personality": The big-five factor structure," Journal of Personality and Social Psychology, vol. 59, no. 6, pp. 1216-1229, 1990.

[11] B. Tversky, "Functional significance of visuospatial representations," in Handbook of higher-level visuospatial thinking, P. Shah and A. Miyake, Eds. Cambridge University Press, 2005, pp. $1-34$.

[12] B. Tversky, M. Agrawala, J. Heiser, P. U. Lee, P. Hanrahan, D. Phan, C. Stolte, and M.-P. Daniele, "Cognitive design principles: From cognitive models to computer models," in Model-based reasoning in science and engineering, L. Magnani, Ed. King's College, 2007, pp. 1-20.

[13] J. Heiser and B. Tversky, "Mental models of complex systems: Structure and function," http://wwwpsych.stanford.edu/simwbt/diagrams/papers/mental_modelsheiser-tversky.pdf.

[14] B. Tversky, J. Zacks, P. U. Lee, and J. Heiser, "Lines, blobs, crosses, and arrows: Diagrammatic communication with schematic figures," in Theory and application of diagrams, M. Anderson, P. Cheng, and V. Haarslev, Eds. Springer, 2000, pp. 221-230.

[15] M. McCaulley, "The myers-briggs type indicator: A jungian model for problem solving," New Directions for Teaching and Learning, vol. 1987, no. 30, pp. 37-53, 1987.

[16] L. Uziel, "The extraverted and the neurotic glasses are of different colors," Personality and Individual Differences, vol. 41, no. 4, pp. 745-754, 2006.

[17] J. Chartrand, M. Rose, T. Elliott, C. Marmarosh, and S. Caldwell, "Peeling back the onion: Personality, problem solving, and career decision-making style correlates of career indecision," Journal of Career Assessment, vol. 1, no. 1, pp. 66-82, 1993
[18] F. H. Farley, "Individual differences in solution time in errorfree problem solving," British Journal of Social and Clinical Psychology, vol. 5, no. 4, pp. 306-309, 1966.

[19] T. Judge, A. Erez, J. Bono, and C. Thoresen, "Are measures of self-esteem, neuroticism, locus of control, and generalized self-efficacy indicators of a common core construct?" Journal of Personality and Social Psychology, vol. 83, no. 3, p. 693, 2002.

[20] J. Walsh, J. Wilding, M. Eysenck, and J. Valentine, "Neuroticism, locus of control, type a behaviour pattern and occupational stress," Work E Stress, vol. 11, no. 2, pp. 148-159, 1997.

[21] J. B. Rotter, "Generalized expectancies for internal versus external control of reinforcement," Psychological Monographs, vol. 80 , no. $609,1966$.

[22] T. A. Judge and J. E. Bono, "Relationship of core selfevaluations traits-self-esteem, generalized self-efficacy, locus of control, and emotional stability-with job satisfaction and job performance: A meta-analysis," Journal of Applied Psychology, vol. 86, no. 1, pp. 80-92, 2001.

[23] M. J. Findley and H. M. Cooper, "Locus of control and academic achievement: A literature review," Journal of Personality and Social Psychology, vol. 44, no. 2, pp. 419-427, 1983.

[24] C. R. Anderson, "Locus of control, coping behaviors, and performance in a stress setting: A longitudinal study," Journal of Applied Psychology, vol. 62, no. 4, pp. 446-451, 1977.

[25] S. Cassidy and P. Eachus, "Learning style, academic belief systems, self-report student proficiency and academic achievement in higher education," Educational Psychology, vol. 20, no. 3, pp. 307-320, 2000

[26] L. R. Goldberg, J. A. Johnson, H. W. Eber, R. Hogan, M. C. Ashton, C. R. Cloninger, and H. C. Gough, "The international personality item pool and the future of public-domain personality measures," Journal of Research in Personality, vol. 40, pp. 84-96, 2006.

[27] J. Heer and M. Bostock, "Crowdsourcing graphical perception: Using Mechanical Turk to assess visualization design," in Proceedings CHI, 2010, pp. 203-212.

[28] R. Kosara and C. Ziemkiewicz, "Do Mechanical Turks dream of square pie charts?" in BELIV Workshop, 2010, pp. 373-382.

[29] A. Kittur, E. H. Chi, and B. Suh, "Crowdsourcing user studies with Mechanical Turk," in Proceedings CHI, 2008, pp. 453-456.

[30] S. Hamlyn-Wright, R. Draghi-Lorenz, and J. Ellis, "Locus of control fails to mediate between stress and anxiety and depression in parents of children with a developmental disorder," Autism, vol. 11, no. 6, pp. 489-501, 2007.

[31] L. M. Lapierre and T. D. Allen, "Control at work, control at home, and planning behavior: Implications for workfamily conflict," 2010, published online before print in Journal of Management.

[32] C. Ziemkiewicz and R. Kosara, "The shaping of information by visual metaphors," IEEE Transactions on Visualization and Computer Graphics, vol. 14, no. 6, pp. 1269-1276, 2008.

[33] National Center for Biotechnology Information, "NCBI genome database," http://www.ncbi.nlm.nih.gov/genome/, accessed 11/18/2010.

[34] M. C. Ioannou, K. Mogg, and B. P. Bradley, "Vigilance for threat: effects of anxiety and defensiveness," Personality and Individual Differences, vol. 36, pp. 1879-1891, 2004.

[35] J. Hollan, E. Hutchins, and D. Kirsh, "Distributed cognition: Toward a new foundation for human-computer interaction research," ACM Transactions on Computer-Human Interaction, vol. 7, no. 2, pp. 174-196, 2000.

[36] Z. Liu, N. Nersessian, and J. Stasko, "Distributed cognition as a theoretical framework for information visualization," IEEE Transactions on Visualization and Computer Graphics, vol. 14, no. 6, pp. 1173-1180, 2008.

[37] E. R. Tufte, The Visual Display of Quantitative Information. Graphics Press, 1983.

[38] J. S. Yi, "Implications of individual differences on evaluating information visualization techniques," in Proceedings of the BELIV Workshop, 2010. 